\title{
Aproximación a las pedagogías emergentes en ambientes virtuales de aprendizaje
}

Approach to emerging pedagogies in virtual learning environments

\author{
Octavio Lecaros Palma ${ }^{1}$ \\ Universidad de Magallanes
}

Recibido: 09.12.2020

Aceptado: 23.12.2020

\section{Resumen}

Este resumen recoge algunos de los temas tratados en el curso de actualización de mi práctica docente "Pedagogías emergentes en ambientes virtuales de aprendizaje", que revisa muchas Metodologías activas para el aprendizaje, Evaluación de estas y algunas sobre Ética, Educación y Tecnología.

En forma especial considera brevemente el uso de las TIC como herramienta cognitiva aplicada a la investigación la que nos exige como docentes un estudio muy acabado de éstas para que los estudiantes puedan disponer de estas amplificadoras de la mente, que estimulen el pensamiento crítico y puedan construir nuevo conocimiento a través de la retroalimentación colaborativa, aún en entornos de incertidumbre muy complejos con una participación social interactiva.

Aprender en tiempos virtuales de aprendizaje es un trabajo extenso pues considera una visión desde Platón, Aristóteles hasta la Inteligencia artificial de nuestros días. El profesor que era el poseedor del conocimiento, hoy en la práctica llega a ser solamente un guía, siempre presente,

\footnotetext{
1 octavio.lecaros@umag.cl

https://orcid.org/0000-0002-9707-5329
} 
que observa, propone y estimula al estudiante a través de nuevas y diferentes tecnologías de aprendizaje, así como también aplica nuevas técnicas de la evaluación de este.

Palabras clave: Pedagogías emergentes, Ambiente virtual, Aprendizaje.

\begin{abstract}
This abstract contains some of the topics covered in the refreshing course on teaching practice "Emerging Pedagogies in Virtual Learning Environments". The reviewed course has many active methodologies for learning and their evaluation. Moreover, it explains some aspects on ethics, education and technology.

In particular, it briefly considers the use of ICT as a cognitive tool applied to research which requires their very detailed study from teachers. They will help students to use them as amplifiers of the mind, that stimulate critical thinking and can build new knowledge through collaborative feedback, even in environments of very complex uncertainty with interactive social participation. Teaching in virtual learning is an extensive job because it considers a vision from Plato, Aristotle to the artificial intelligence of our day. The teacher who was in the past the knowledge owner, today in practice, he becomes only an always present guide. He observes and stimulates students proposing and applying new different learning technologies.
\end{abstract}

Keywords: Emerging-pedagogies, Virtual-environment, Learning.

\title{
1. Pedagogías emergentes en ambientes virtuales de aprendizaje
}

El revolucionario transitar desde una cultura agrícola a otra superior, destacada por los cambios sociales, económicos y culturales originando transformaciones industriales, innovadoras en la producción resultante de esta nueva relación: mecánico -energía-vaporagua. (XVIII) responsable de una revolución industrial-eléctrica. Esta condujo a la 
automatización, nuevas tecnologías, las TICs, (Aparicio Gómez, 2019), interconexión, luz, TV, desde la Web 1.0 (1980-90), red multimedia, hasta la inteligencia artificial 4.0. (2010). (Demartini, C y Benussi, L 2017).

Aprender con Big Data, (Yuan, 2019), web social, computación en la nube, etc., producen un florecimiento de la educación, cambian las (TAC) de Tecnología del aprendizaje y la comunicación a las (TEP) para el empoderamiento y la participación.

El Aprendizaje Continuo que requiere aprender con las Tecnologías para toda la vida. Con softwares especiales en aulas virtuales e interpretación de datos. Siempre conlleva acompañamiento, un aprendizaje compartido, integrado, interdisciplinario, socializado con entornos significativos en tiempos de incertidumbre. Estrategias virtuales, prácticas innovadoras con enfoques en ideas no sistematizadas, aulas invertidas centrada ahora según el lugar que interese al estudiante donde el profesor ahora es sólo un acompañante. Evaluación de la Web, el e-learning, móvil-learning, radio, TV, es propia de una educación abierta a distancia y conforme estas llegan, (teorías emergentes) se usan en contextos significativos.

\section{Metodologías activas}

La Innovación patenta resultados por gestión de hallazgos. El profesor siempre presente para generar entusiasmo. Muchas metodologías emergen, y permiten probar, experimentar con autoaprendizaje y conocimiento.

Aprender a través de juegos: Gamificación (Clark et al., 2016) señala que ésta, en general, ha sido parte de la vida en los últimos 30 años, consolidando el desafío del hasta donde puedo llegar; retroalimentación, persistencia en los videos-juego con entornos difíciles, complicados, en ambientes virtuales con modelamientos, son escenarios, que nos enseñan y alfabetizan digitalmente. Aprendizaje con tecnología basado en proyectos, en diseños, mezclando aprendizaje y diversión promoviendo creatividad, desarrollan habilidades, las socializan, motivan para desarrollar capacidades futuras, con oportunidad de conocer sus límites. El 
Flipped Learning. (Gnutova, 2020) para el acompañamiento y la independencia donde puede ser acompañado por la familia, amigos, compañeros de clases, retroalimentados por profesores adecuados, profundizan el tema, generando nuevo conocimiento. Aprendizaje invertido en casa y en el aula. El Design Thinking (Artiles, J. A \& Lande, 2016) para resolver problemas la Alfabetización Visual, con diagramas, movimientos de imágenes, esquemas. El Teachback (Dinh, T.T.H ${ }^{14}$ et al., 2016) para aprender a través de la conversación estructurada. El Social Media (De Hart, J., Stell, M., \& Grant, C 2020) en las redes sociales, comparten fotos, manejo de dinero, videos conferencias, descargan libros, leen noticias, compran bienes en tiempo real.

\section{Evaluación y actualización}

Las Analíticas de Aprendizaje importan para conocer cómo progresan los estudiantes respecto a los objetivos propuestos, buscando referente, medirlos, mejorarlos y perfeccionarlos. Preguntándose siempre ¿por qué analizo lo que analizo?, las dificultades y entornos del aprendizaje y utilidad de los materiales. Hacer análisis comparativos con los demás y hallar indicadores de éxito o de abandono y los procesos que le permiten aprender. Proporcionar apoyo digital extra, con softwares especializados con retroalimentación y seguimiento.

El Blockchain. (Sharples, M. \& Domingue, J. 2016), nueva tecnología digital, con registro educativos, textos imágenes, diplomas, softwares vinculados entre sí. Permite una gestión expedita, con artículos educativos; con registro público, cadenas de bloques que contienen todos los temas educativos, creaciones literarias. Poseen base de datos de gran confianza digital.

El Stealth Assesment, (Geordiadis. $\mathbf{K}^{\mathbf{6}}$ et. al. 2019), (Shute, 2011). Evaluación Discreta mide el apoyo de la Enseñanza con videos juegos. Sigue el progreso de la persona en su proceso evaluativo. Acompaña al proceso educativo en las simulaciones, con monitoreo dinámico, evalúa el aprendizaje por competencia y se retroalimenta con narrativas del juego y simulaciones del entorno. Es una Evaluación con softwares especiales con tecnología de procesos, modelos de rendimientos, gráficos de barras, etc. insignias que acredita lo logrado, 
el scouting para usar con orgullo. Muestra continuamente el progreso del proceso educativo y evaluación de una persona.

\section{Acción actualización de la práctica docente}

La Ética de la reflexión profunda sobre la moral habitual, perfeccionar el ejercicio del pensar, de ser críticos al pensar por uno mismo, en la interiorización del conocimiento (Kohlberg).

Análisis de las emociones: (O’Reilly, 2015) determinante y clave para el aprendizaje. Usan Inteligencia artificial, hacen estudios de mercado a través de las cámaras, con una intromisión con los anuncios propagandas, con tecnologías complejas, nos observan como los movimientos de ojos muestran intereses.

El Aprendizaje a través de la Argumentación, permite a estudiantes y profesores esperar respuestas conocidas, lo contrario. Argumentación oportuna frente a preguntas no hechas.

Aprendizaje para el futuro. Prepara al estudiante para el trabajo en un entorno muy complejo, para la incertidumbre, conectando el aprendizaje formal con el mundo real, preparándolo para lo que no sabe. Requiere de una pedagogía especial centrada en aprender y reaprender. Nuevas tecnologías con soportes de retroalimentación, fuera del aula, frente a un futuro impredecible.

Aprendizaje con valores internos, como el Evo Room, de simulaciones inmersivas de un ecosistema Tropical (Lui, M. y Slotta, 2013), la plataforma de aprendizaje digital basada en la investigación. El aprendizaje es significativo quizás considera lo previo para construir lo posterior. Es como un andamio de construir teniendo claro cuál es el valor que, se quiere conseguir. La Inteligencia artificial (Sánchez-Maroño, et al., 2014), permite interacción en los contenidos; la plataforma de red, ejemplo Moodle que admite gran interacción entre personas, nuevos entornos de aprendizaje usando nuevas tecnologías.

Volumen 1. Número 2. Julio - Diciembre 2021 


\section{Conclusiones}

El Aprendizaje para el futuro exige "pedagogías innovadoras", novedosas, cambiantes en la enseñanza- aprendizaje para el mundo moderno, habilitado por la tecnología como las TIC, que son verdaderas amplificadoras y organizadoras de la mente, verdaderos andamios cognitivos, que empoderan a profesores y alumnos transformando los contenidos conducentes hacia una actualización permanente.

La Gamificación, consolas (1980) a través de juego ha sido parte de la vida consolidando el desafío, serán inductores permanentes de la persistencia de escenarios y ambientes virtuales o el Flipped Learning acompañado por la familia, amigos, compañeros, provocando sin duda un aprendizaje invertido.

Al evaluar los aprendizajes podría considerarse que el Stealth Assesment o Evaluación Discreta al seguir continuamente el progreso de una persona en su proceso evaluativo, a través de softwares de juegos y/o simulaciones dinámicas, será cada vez más importante, como simulaciones dinámicas de ambientes tropicales traídos al aula, donde los estudiantes observarán sin darse cuenta de su evaluación.

Las nuevas tecnologías para el aprendizaje requerirán siempre de facilitadores preparados como "profesores": que enseñen con herramientas cognitivas, comprometidos en el oficio de sabio: Indagar, investigar, innovar, por lo que deberán acompañar al discípulo, empleando las TIC desde la educación pre - escolar, la educación básica primaria y secundaria, con acompañamiento de padres, profesores y sus pares, hasta los procesos de apropiación en la Educación Superior que necesitará innovación educativa continua y gestión de conocimiento para el profesorado al usar las TIC, puesto que la Universidad, al producir conocimiento con la apropiación de la tecnología generará continuamente nuevos procesos.

A pesar del empleo de "simulaciones, (Evo Room), las TIC todavía no logran reemplazar lo experimental, como son las experiencias químicas complejas, multifacéticas, de logros 
estrictamente vivenciales, que otorgan habilidades personales, destrezas, obtenidas sólo presencialmente en aula (Laboratorio de Química), observación científica, trabajo con respeto al medio ambiente, menos aún en tiempos de Pandemia.

\section{Referencias}

Aparicio Gómez, O. Y. (2019). Uso y apropiación de las TIC en educación. Revista Interamericana de Investigación, Educación y Pedagogía, RIIEP. https://doi.org/10.15332/s1657-107x.2019.0001.04

Aparicio Gómez, O. Y., \& Ostos Ortiz, O. L. (2018b). Las TIC como herramientas cognitivas para la investigación. Revista Interamericana de Investigación, Educación y Pedagogía, RIIEP, 11(1), 81-86. https://doi.org/10.15332/s1657-107x.2018.0001.08

Aparicio-Gómez, Oscar-Yecid (2017). Uso de las TIC en el Colegio Padre Manyanet - Chía, Colombia (2011-2014). En Aparicio-Gómez, Oscar-Yecid y Aparicio-Gómez, WilliamOswaldo (Eds.). Uso de las TIC para la innovación (pp. 129-158). Bogotá, Colombia: Ed\&TIC. ISBN: 978-958-26-0367-0.

Aparicio-Gómez, Oscar-Yecid (2020a). El oficio de sabio: Indagar, investigar, innovar. En: Aparicio-Gómez, Oscar-Yecid y Ostos-Ortiz, Olga-Lucía. (Eds.). Innovación Educativa y Gestión del Conocimiento (pp. 177-192). Bogotá, Colombia: Universidad Santo Tomás. ISBN: 978-958-782-304-2

Aparicio-Gómez, William-Oswaldo y Aparicio-Gómez Oscar-Yecid (2020). Aprendizaje basado en problemas y uso de las TIC. Working Paper No. 200140. DOI: 10.13140/RG.2.2.20238.79681

Aparicio-Gómez, William-Oswaldo y Aparicio-Gómez Oscar-Yecid (2020). Evaluación en entornos digitales. Working Paper No. 200147. DOI: 10.13140/RG.2.2.26399.18080 
Artiles, J. A., \& Lande, M. (2016). Broadening non-designers'solutions for big issues: The Education Design Shop design thinking workshop. International Journal of Engineering Education, 32(3), 1418-1427. Tempus Publications.

Bennett, J., \& Lubben F. (2006). Context-based chemistry: theSalters approach. International Journal of Science Education, 28(9), 999-1015.

Buchanan, R. (1992). Wicked problems in design thinking. Design Issues, 8(2), 5-21.

Clark, D.B., Tanner-Smith, E.E. \& Killingsworth, S. S. (2016). Digital games, design, and learning: a systematic review and meta-analysis. Review of Educational Research, 86(1), 79122

DeHart, J., Stell, M., \& Grant, C. (2020). Social media and the scourge of visual privacy. Information (Switzerland), 11(2). https://doi.org/10.3390/info11020057

Dori, Y. J., Avargil, S., Kohen, Z., \& Saar, L. (2018). Context-based learning and metacognitive prompts for enhancing scientific text comprehension. International Journal of Science Education, 40(10), 1198-1220. https://doi.org/10.1080/09500693.2018.1470351

Demartini, C., y Benussi, L. (2017). Do Web 4.0 and Industry 4.0 Imply Education X.0? IT Pro May/June 2017, IEEE Computer Society.

Dinh, T.T.H., Bonner, A., Clark, R. Ramsbotham, J. \& Hines, S. (2016). The effectiveness of the teach-back method on adherence and self-management in health education for people with chronic disease: a systematic review. JBI Database of Systematic Reviews and Implementation Reports, 14(1), 210-247.

Finzer, W. (2013). The data science education dilemma. Technology Innovations in Statistics Education, 
Georgiadis, K., Van Lankveld, G., Bahreini, K., \& Westera, W. (2019). Learning analytics should analyse the learning: Proposing a generic stealth assessment tool. IEEE Conference on Computatonal Intelligence and Games, CIG, 2019-August. https://doi.org/10.1109/CIG.2019.8847960

Gnutova, I. I. (2020). From flipped classroom to flipped learning: Evolution of the concept and its philosophical foundations. Vysshee Obrazovanie v Rossii, 29(3), 86-95. https://doi.org/10.31992/0869-3617-2020-29-3-86-95

Mahmud, S. N. D., Husnin, H., \& Soh, T. M. T. (2020). Teaching presence in online gamified education for sustainability learning. Sustainability (Switzerland), 12(9). https://doi.org/10.3390/su12093801

O'Reilly, M. (2015). The influence of emotions, attitudes and perceptions on learning with technology. Research in Learning Technology, Vol. 23. https://doi.org/10.3402/rlt.v23.27763

Quintana J., Aparicio-Gómez, Oscar-Yecid (Eds.) (2017). Temas emergentes en educación. Bogotá, Colombia: Universidad Central. ISBN: 978-958-26-0367-0.

Sánchez-Maroño, N., Alonso-Betanzos, A., Fontenla-Romero, O., Brinquis-Núñez, C., Polhill, J. G., \& Craig, T. (2014). Influence of internal values and social networks for achieving sustainable organizations. Frontiers in Artificial Intelligence and Applications, 263, 1179-1184. https://doi.org/10.3233/978-1-61499-419-0-1179

Sharples, M. \& Domingue, J. (2016). The Blockchain and Kudos: a distributed system for educational record, reputation and reward. In K. Verbert, M. Sharples \& T. Klobučar (Eds.) Adaptive and Adaptable Learning: Proceedings of 11th European Conference on Technology Enhanced Learning (EC-TEL 2015), Lyon, France, 13-16 September 2016. Switzerland: Springer International Publishing, 490-496. 
Shute, V. J. (2011). Stealth Assessment in computer-based games to support learning. In S. Tobias and J. D. Fletcher (Eds.) Computer Games and Instruction, Charlotte: Information Age Publishing. 503-524.

Yuan, L. (2019). Discussion on the application of computer big data in internet learning. Proceedings - 2019 12th International Conference on Intelligent Computation Technology and Automation, ICICTA 2019, 482-486. https://doi.org/10.1109/ICICTA49267.2019.00109

Lui, M. y Slotta, JD (2013). Simulaciones inmersivas para aulas inteligentes: exploración de conceptos evolutivos en ciencia secundaria, tecnología, pedagogía y educación, DOI: 10.1080 / 1475939X.2013.838452

Lui, M. y Slotta, JD (2013). Explorando conceptos evolutivos con simulaciones inmersivas. Actas de la X Conferencia Internacional de Ciencias del Aprendizaje (CSCL) - Volumen 1, (págs. 304-311) Sociedad Internacional de Ciencias del Aprendizaje (ISLS). 